\title{
Transthoracic needle biopsy of thoracic tumours by a colour Doppler ultrasound puncture guiding device
}

Hao-Chien Wang, Chong-Jen Yu, Dun-Bing Chang, Ang Yuan, Yuan-Chie Lee, Pan-Chyr Yang, Sow-Hsong Kuo, Kwen-Tay Luh

\begin{abstract}
Background - Ultrasound guided transthoracic needle aspiration biopsy has recently been used to obtain specimens for histological diagnosis of pulmonary and mediastinal tumours. Conventional real time, grey scale puncture guiding devices cannot differentiate vascular structures, and clear visualisation of the needle shaft or tip within a desired target is not always possible. This study describes a new builtin colour Doppler ultrasound puncture guiding device and assesses the relative safety of transthoracic needle aspiration biopsy of thoracic tumours by grey scale or colour Doppler ultrasound guidance. Methods - Thirty patients with radiographic evidence of pulmonary (22 patients) or mediastinal tumours (eight patients) underwent ultrasonographic evaluation and transthoracic needle aspiration biopsy by using the colour Doppler ultrasound puncture guiding device (Aloka UST 5045P-3.5). These tumours were initially examined by grey scale ultrasound, and colour Doppler imaging was then used to evaluate the number of blood vessels surrounding and within the target tumour and the possibility of visualisation of the needle shaft or needle tip during the aspiration biopsy procedure.
\end{abstract}

Results - The colour Doppler ultrasound guiding device was far superior to the grey scale device for identification of the number of vessels surrounding or within the target tumour (83\% v 20\%) and for visualisation of the needle shaft or needle tip $(80 \%$ v $17 \%)$.

Conclusions - By using the colour Doppler ultrasound puncture device, vascular structures surrounding or within the target tumour can be verified. Visualisation of the needle shaft or tip is also better. Biopsy routes can be selected to avoid puncturing vessels. This approach should be particularly helpful for guiding biopsies of mediastinal tumours, where puncturing the heart or great vessels is a potential complication.

(Thorax 1995;50:1258-1263)

Keywords: colour Doppler ultrasound, ultrasound puncture guiding device, transthoracic biopsy.
Percutaneous transthoracic needle aspiration biopsy under ultrasound guidance is a sensitive and accurate approach used to obtain specimens for histological diagnosis of pulmonary tumours. ${ }^{1-8}$ The conventional real time, grey scale guiding device has a complication rate of about $0-8 \%$ which includes haemoptysis and pneumothorax. ${ }^{28-13}$ Complications occur mainly from inadvertent puncture of vessels and aerated lung. These devices are able to detect vascular structures surrounding or within the target lesion by demonstrating anechoic tubular structures, further confirmed by pulse wave analysis with duplex Doppler. However, for small vessels and deep seated lesions the acquisition of vascular tubular images may not be possible. Puncturing of these vascular structures under grey scale ultrasound guidance still occasionally occurs. It has been reported that fine needle transthoracic aspiration with a gauge 20 or 21 needle is relatively safe even when puncturing a great vessel. ${ }^{9}$ However, cutting a biopsy specimen with a large bore Trucut needle may still be risky, particularly in mediastinal tumours where tissue biopsy is nearly always necessary. ${ }^{31114}$

The recent advent of colour Doppler imaging has greatly improved the visualisation of vascular structures and can demonstrate the tumour vessels. ${ }^{15}$ It has been shown that colour Doppler imaging can enhance visualisation of the needle tip and shaft during aspiration biopsy. ${ }^{1617}$ Colour Doppler ultrasound guidance should provide a more detailed image and reduce the risk of conducting a transthoracic biopsy. This study describes a new built-in colour Doppler ultrasound guiding device, and compares the relative safety of transthoracic needle aspiration biopsy of thoracic tumours under grey scale and colour Doppler ultrasound guidance.

\section{Methods}

Thirty consecutive patients with radiographic evidence of a thoracic tumour underwent chest ultrasonographic examination at the National Taiwan University Hospital between October 1993 and February 1994. They were selected for ultrasound guided transthoracic aspiration biopsy based on the following criteria: (1) mediastinal or pulmonary tumours with an accessible ultrasound window; (2) no definite histological diagnosis after bronchoscopy or sputum cytological examination; (3) no bleeding tendency (platelet count of $>100000 / \mu \mathrm{l}$ 


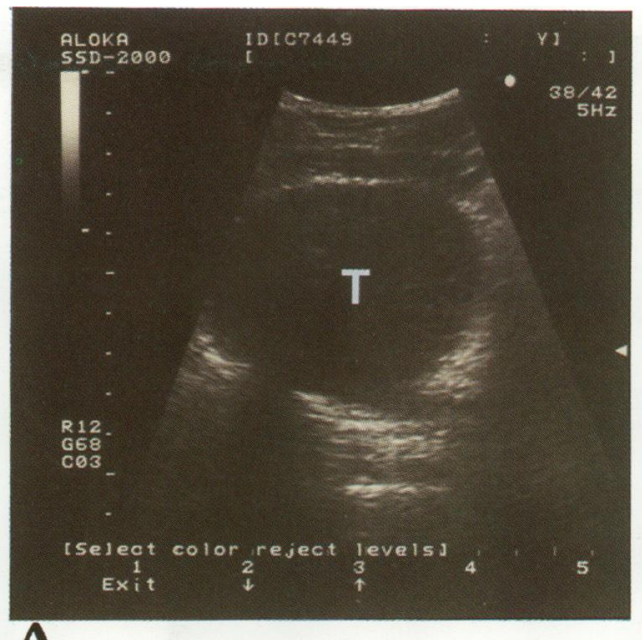

A
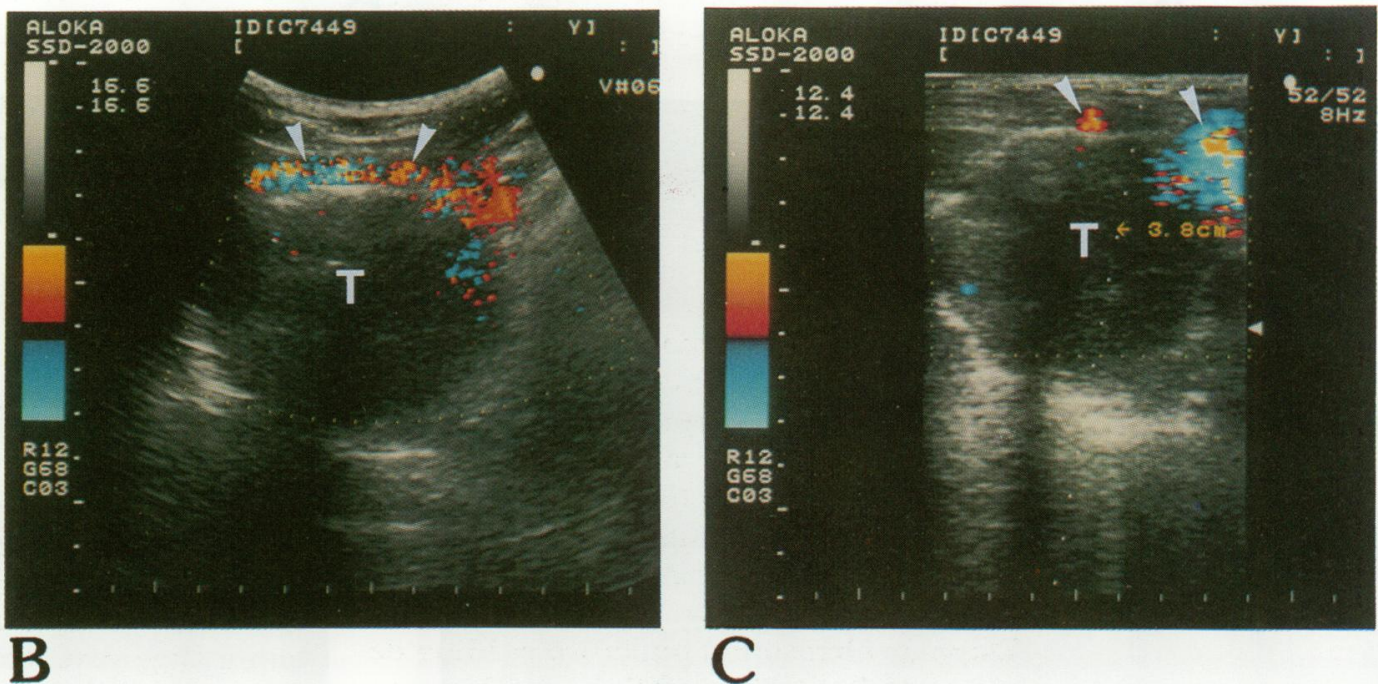

Figure 1 (A) A 36 year old woman with a pulmonary tumour at the right apex; the grey scale ultrasound image reveals no definite blood vessel surrounding or within it. (B) The same tumour evaluated with colour Doppler ultrasound shows an artery (arrow head) just overlying the tumour. (C) The direction of puncture probe can be adjusted from $-5^{\circ}$ to $20^{\circ}$ to preselect a puncture route and depth (yellow dotted line and arrow) to avoid puncturing the vessels (arrow head). $T=$ tumour.

and normal prothrombin times); (4) consent to receive the procedure and were cooperative. The ultrasound examinations were performed with real time, linear, and convex ultrasound units with a $3.5 \mathrm{MHz}$ transducer (Aloka SSD 2000, Aloka, Tokyo). This machine allows both grey scale and colour Doppler ultrasound imaging by using a colour Doppler mode button. Two different pairs of operators, using the same machine, performed either the grey scale or colour Doppler ultrasound study and ultrasound guided biopsy independently. The examination of the thoracic tumour was initially done with real time, grey scale ultrasound, then followed by colour Doppler imaging. The following data were obtained: (1) both longitudinal and transverse images to assess the two dimensional configuration; and (2) the number of blood vessels within $1 \mathrm{~cm}$ of the target tumour and those within the target tumour. The colour sensitivity setting was adjusted to a minimal range of $+5 \mathrm{~cm} / \mathrm{s}$. To avoid false positive vessel visualisation, the tubular structures detected by grey scale ultrasound and colour Doppler flow signals were further confirmed to be vascular structures by spectral wave analysis.

After the initial evaluation the skin was disinfected and anaesthetised. A sterile colour puncture transducer (Aloka UST 5045P-3.5, Aloka, Tokyo, Japan) equipped with a guiding channel was applied. This puncture probe also possesses both grey scale and colour Doppler imaging. The aspiration biopsy was performed twice by different pairs of operators either by grey scale or colour Doppler imaging.

Figure 1 illustrates how the puncture route is selected while conducting a transthoracic biopsy. This ultrasound guided aspiration biopsy technique has been reported in previous studies. ${ }^{18-20}$ The puncture needle used was a 22 gauge Chiba needle made of steel and composed of an inner stylet with scored outer sheath. The patient was asked to hold his/her breath while the needle was inserted through the guiding channel, and aspiration biopsy was performed. The cytological specimen was stained with Riu's method and interpreted immediately. ${ }^{21}$ Patients with a mediastinal tumour or pulmonary tumour without a definite histo- 

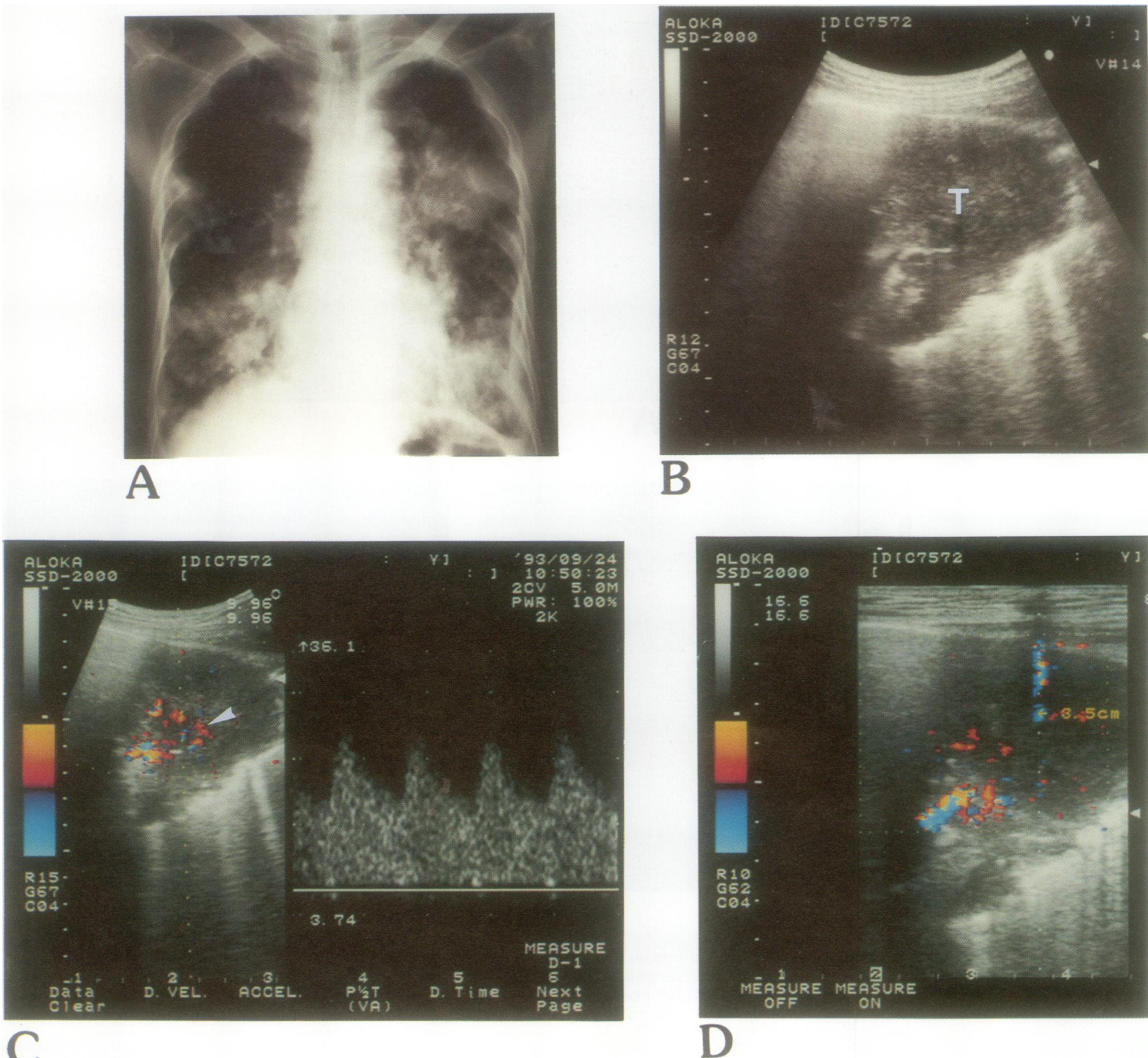

Figure $2(A)$ Chest radiograph of a 73 year old man showing multiple pulmonary tumours. (B) The grey scale image of a left upper lobe tumour which fails to demonstrate any vessel. (C) Many vessels (arrow heads) are identified within the tumour by colour Doppler imaging. (D) Colour Doppler ultrasound image indicating the needle shaft and tip (arrow).

logical diagnosis after needle aspiration cytological examination underwent cutting biopsies twice by both grey scale and colour Doppler imaging with a gauge 16 Trucut needle (Top Surgical, Tokyo, Japan).

The two operators were asked whether the needle shaft or tip could be seen during the procedure. They appear as a whitish spot in grey scale ultrasound ${ }^{2}$ while, with colour Doppler ultrasound, a clear linear colour marking is seen (figs 2 and 3 ). The process of guided biopsy required 10-15 seconds to be completed. A video cassette recorder was used during the procedure to confirm the result. After needle aspiration biopsy, any possible complication was observed.

Statistical analysis was conducted using McNemar's test.

\section{Results}

Thirty patients ( 18 men) of mean age 51 years (range 19-78) were included in the study. The size of the tumours ranged from $1.6 \times 1.9$ to $9.0 \times 11.4 \mathrm{~cm}$. Thirty patients underwent needle aspiration biopsy and 13 patients underwent additional cutting biopsy with a Trucut needle. The demographic data are shown in table 1 . The blood vessels surrounding and within the target tumour were identified by grey scale imaging in six patients $(20 \%)$ with a total number of eight vessels (table 2). By colour Doppler imaging, blood vessels surrounding and within the target tumour were identified in 25 patients $(83 \%)$, and eight tumours showed more than 10 blood vessels. No obvious vascular structure was identified by either method of image analysis in five patients. 

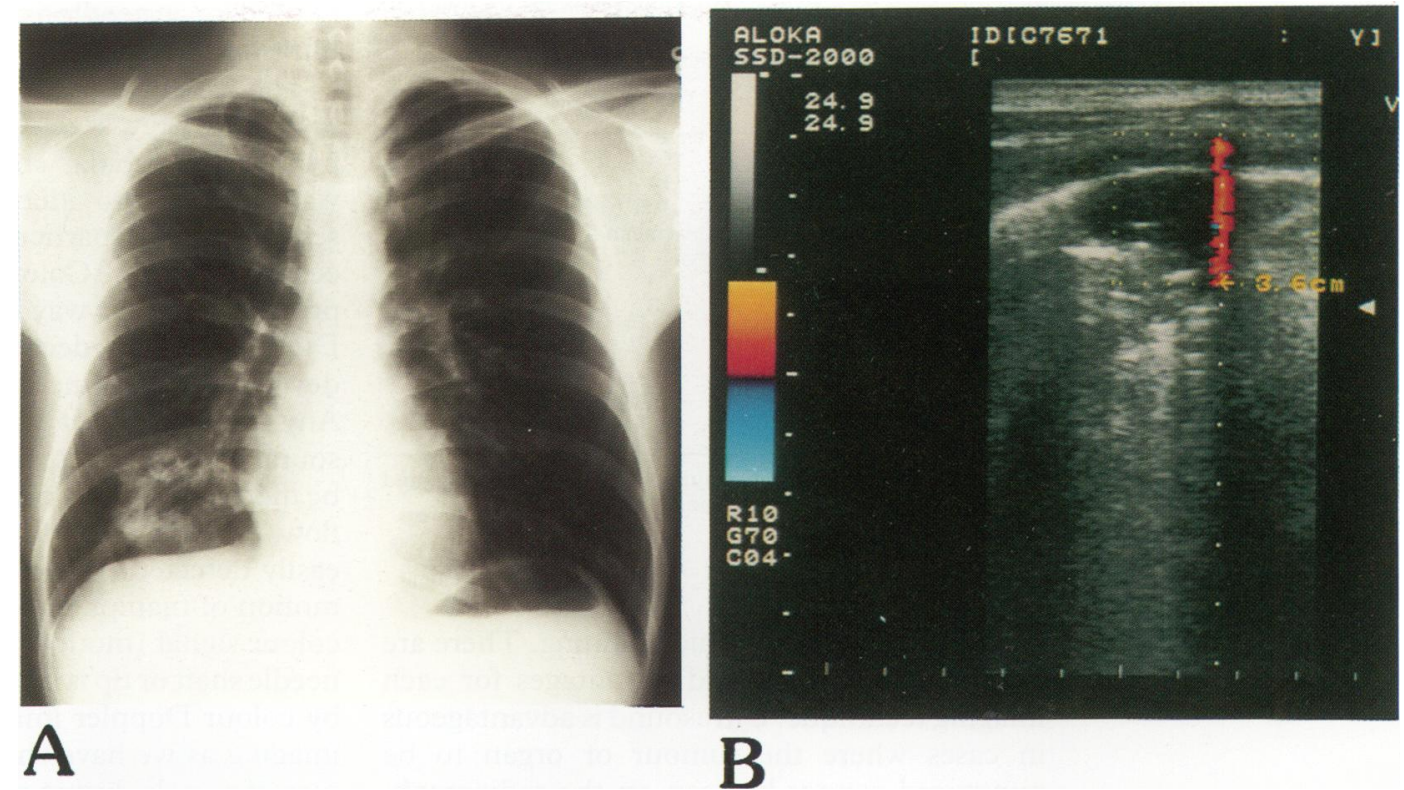

Figure 3 (A) Chest radiograph of a 31 year old man showing a cavitary mass in the right lower lobe. (B) Colour Doppler image showing the needle shaft and tip (arrow) during aspiration biopsy.

Table 1 Results of ultrasound guided aspiraton biopsy of 30 patients with thoracic tumour

\begin{tabular}{|c|c|c|c|}
\hline \multirow[t]{2}{*}{ Caselage/sex } & \multirow[t]{2}{*}{ Tumour size $(\mathrm{cm})$} & \multicolumn{2}{|l|}{ Cytological and histological results } \\
\hline & & Grey scale & Colour Doppler \\
\hline \multicolumn{4}{|l|}{ Cytology: } \\
\hline \multicolumn{4}{|c|}{ Mediastinal tumour } \\
\hline $1 / 19 / \mathrm{M}$ & $5.5 \times 4.0$ & Inadequate specimen* & Inadequate specimen* \\
\hline $2 / 27 / \mathrm{F}$ & $3.4 \times 3.4$ & Lymphoma & Lymphoma \\
\hline $3 / 43 / \mathrm{F}$ & $5.0 \times 3.0$ & Blood only & Squamous cell carcinoma \\
\hline \multicolumn{4}{|c|}{ Pulmonary tumour } \\
\hline $4 / 68 / \mathrm{M}$ & $4 \cdot 8 \times 7 \cdot 1$ & Squamous cell carcinoma & Squamous cell carcinoma \\
\hline $5 / 58 / \mathrm{F}$ & $6.5 \times 7.0$ & Tuberculosis & Tuberculosis \\
\hline 6/73/M & $6.5 \times 7.0$ & Squamous cell carcinoma & Squamous cell carcinoma \\
\hline $7 / 54 / \mathrm{M}$ & $2 \cdot 2 \times 1.7$ & Blood only & Adenocarcinoma \\
\hline $8 / 49 / \mathrm{M}$ & $2 \cdot 0 \times 2 \cdot 0$ & Adenocarcinoma & Adenocarcinoma \\
\hline 9/43/M & $3.8 \times 2.5$ & Tuberculosis & Tuberculosis \\
\hline $10 / 78 / F$ & $4.5 \times 5.0$ & Squamous cell carcinoma & Squamous cell carcinoma \\
\hline $11 / / 36 / \mathrm{F}$ & $6.0 \times 6.5$ & Adenocarcinoma & Adenocarcinoma \\
\hline $12 / 58 / \mathrm{M}$ & $4.3 \times 4.8$ & Blood only & Small cell carcinoma \\
\hline $13 / 60 / \mathrm{M}$ & $8.5 \times 10.0$ & Squamous cell carcinoma & Squamous cell carcinoma \\
\hline $14 / 51 / \mathrm{M}$ & $1.6 \times 1.9$ & Squamous cell carcinoma & Squamous cell carcinoma \\
\hline $15 / 67 / \mathrm{M}$ & $7.5 \times 6.0$ & Squamous cell carcinoma & Squamous cell carcinoma \\
\hline $16 / 68 / \mathrm{M}$ & $9.0 \times 9.0$ & Squamous cell carcinoma & Squamous cell carcinoma \\
\hline $17 / 51 / \mathrm{F}$ & $5.0 \times 5.0$ & Fibrosis & Fibrosis \\
\hline \\
\hline \multicolumn{4}{|c|}{ Mediastinal tumour } \\
\hline $18 / 29 / \mathrm{F}$ & $5.3 \times 6.0$ & Lymphoma & Lymphoma \\
\hline $19 / 41 / \mathrm{M}$ & $3.8 \times 4.6$ & NPC metastasis & NPC metastasis \\
\hline $20 / 28 / \mathrm{F}$ & $3.0 \times 2.7$ & Poorly differentiated carcinoma & Poorly differentiated carcinoma \\
\hline $21 / 37 / M$ & $3.1 \times 3 \cdot 1$ & Small cell carcinoma & Small cell carcinoma \\
\hline $22 / 74 / \mathrm{M}$ & $3.0 \times 4.0$ & Thymic carcinoma & Thymic carcinoma \\
\hline \multicolumn{4}{|c|}{ Pulmonary tumour } \\
\hline $23 / 31 / M$ & $4.7 \times 1.7$ & Chronic inflammation & Chronic inflammation \\
\hline $24 / 54 / M$ & $9.0 \times 11.4$ & Squamous cell carcinoma & Squamous cell carcinoma \\
\hline $25 / 71 / \mathrm{F}$ & $6.0 \times 7.0$ & Squamous cell carcinoma & Squamous cell carcinoma \\
\hline $26 / 36 / F$ & $3.2 \times 3.6$ & Hibernoma & Hibernoma \\
\hline $27 / 36 / \mathrm{F}$ & $5.9 \times 7.3$ & Chronic inflammation & Chronic inflammation \\
\hline $28 / 68 / M$ & $7.5 \times 7.9$ & NPC metastasis & NPC metastasis \\
\hline $29 / 53 / M$ & $3.0 \times 5.0$ & Squamous cell carcinoma & Squamous cell carcinoma \\
\hline $30 / 54 / \mathrm{F}$ & $2.0 \times 1.9$ & Tuberculosis & Tuberculosis \\
\hline
\end{tabular}

*Aspiration result showed benign cells but patient refused cutting biopsy.

NPC $=$ nasopharyngeal carcinoma.

Colour Doppler imaging was able to identify vessels surrounding and within 15 tumours, which were not identified by grey scale imaging. The needle shaft or tip was identified by grey scale imaging during the procedure of aspiration biopsy in only five patients (17\%), whereas in 23 patients $(80 \%)$ it was identified by colour Doppler imaging. No complications including pneumothorax or haemoptysis were found.

In three cases (one mediastinal tumour and two pulmonary tumours) specimens obtained under colour Doppler imaging gave a precise diagnosis, whereas samples under grey scale imaging were non-diagnostic. The diagnostic yield of cutting biopsy samples (table 1) was identical for both imaging techniques.

\section{Discussion}

Percutaneous puncture to obtain specimens for histological or microbiological study is a well established procedure. ${ }^{1}$ It can be performed under the guidance of fluoroscopy, ultrasound, 
Table 2 Results of guided biopsy with grey scale versus colour Doppler image analyses in 30 patients with thoracic tumours

\begin{tabular}{|c|c|c|}
\hline & $\begin{array}{l}\text { Mediastinal tumour } \\
\quad(n=8)\end{array}$ & $\begin{array}{l}\text { Pulmonary tumour } \\
(n=22)\end{array}$ \\
\hline $\begin{array}{l}\text { Mean age (years) } \\
\text { Sex }(M / F) \\
\text { Tumour diameter }(\mathrm{cm})\end{array}$ & $\begin{array}{l}37 \\
4 / 4 \\
2 \cdot 9-5 \cdot 7 \\
(\text { mean } 4 \cdot 0)\end{array}$ & $\begin{array}{l}55 \\
14 / 8 \\
1 \cdot 8-10 \cdot 2 \\
(\text { mean } 5 \cdot 2)\end{array}$ \\
\hline $\begin{array}{l}\text { Grey scale imaging } \\
\text { Needle tip visualised } \\
\text { Vessels visualised* } \\
\text { Complications } \\
\text { Colour Doppler imaging } \\
\text { Needle tip visualised } \\
\text { Vessels visualised* } \\
\text { Complications }\end{array}$ & $\begin{array}{c}2 \\
3 \\
\text { Nil } \\
7 \\
7 \\
\text { Nil }\end{array}$ & $\begin{array}{l}3 \\
3 \\
\text { Nil } \\
\\
16^{* *} \\
18^{* *} \\
\text { Nil }\end{array}$ \\
\hline
\end{tabular}

* An average of 1.3 vessels per tumour was visualised in six tumours by grey scale imaging, and of $5 \cdot 2$ vessels per tumour in 25 tumours by colour Doppler imaging.

$* * \mathrm{p}=0.000$ grey scale versus colour Doppler imaging.

or computed tomographic scanning. There are different indications and advantages for each imaging technique. Ultrasound is advantageous in cases where the tumour or organ to be punctured cannot be seen on the radiograph, if it is to be approached from an unusual angle, or where radiography is contraindicated. ${ }^{1}$ The value of real time ultrasound in guiding the needle biopsy in abdominal disease is well documented. ${ }^{18-22}$ Ultrasonography was previously thought to be of limited use in the diagnosis of chest disease because of interference from ribs or aerated lung. ${ }^{1023}$ However, an ultrasound window can be created by atelectasis or non-aerated lung, and allows the study of underlying tumour. ${ }^{7}$ With improved ultrasound resolution and real time monitoring of aspiration biopsy procedures, this modality has proved to be useful for the diagnosis of thoracic lesions. ${ }^{128-13}$

Previous studies of transthoracic needle aspiration biopsy of thoracic tumour under grey scale ultrasound guidance were complicated by haemoptysis and pneumothorax, with a complication rate of $0-8 \% .{ }^{28-13} \mathrm{~A}$ conventional real time, grey scale guiding device cannot always differentiate vascular structures, and exact visualisation of the needle shaft or tip within a desired target is not always possible. There are two ways to improve safety during the puncture procedure: (1) visualisation of the needle shaft or tip; and (2) identification of blood vessels surrounding and within the target tumour. For grey scale, real time ultrasound guidance, needle visualisation can be aided by several methods including roughening or scoring the outer needle or inner stylet and placement of a guide wire through the needle. ${ }^{172425}$ Injection of a tiny amount of saline or air as a contrast marker also improves visualisation of the tip. ${ }^{172425}$ Although the Chiba needle we used was scored (that is, the outer needle has circumferential marks every centimetre to improve the echogenicity), in this study only in $17 \%$ of cases was the needle tip visible on the grey scale image. The needle shaft was also not able to be seen as in previous studies. ${ }^{17}$ The time available for transthoracic aspiration biopsy makes the injection of saline or air impractical because the patient might breathe and hence increase the risk of a complication. Visualisation of the needle tip can be difficult at times, especially if a narrow angle technique is used or a needle is inserted parallel to the ultrasound beam. ${ }^{17}$

The characteristic image of vessels under real time, grey scale ultrasound is a pulsatile hypoechoic tubular structure. Hence, it is always difficult to identify vessels under grey scale imaging, particularly within the hypoechoic tumour. Colour Doppler ultrasound provides another way of solving this problem. During the past decade colour Doppler flow devices have become available and widely used. Any moving object will emit or reflect ultrasound which creates a Doppler shift. ${ }^{26}$ This can be modulated into a colour signal which mimics flow. With this technique, blood vessels can be easily detected. When a needle is inserted the motion of manipulation will produce the same colour signal (motion marking) ${ }^{1617}$ Hence the needle shaft or tip is much more easily identified by colour Doppler imaging than by grey scale imaging as we have shown. Inadvertent puncture of vessels during aspiration may dilute the tissue in the needle and decrease diagnostic yield.

Ultrasound guided fine needle aspiration of mediastinal tumours has a low sensitivity for precise diagnosis because of pleomorphic histology. ${ }^{3114}$ This can be improved by core biopsy with a Trucut needle. ${ }^{311}$ Puncturing great vessels or even cutting the vessels within the mediastinum can cause serious complications. The colour Doppler guiding device should allow cutting needle biopsies to be performed even more safely, although we experienced no complications with either technique in this study and yields were identical.

Three types of ultrasound guiding devices are available, ${ }^{11}$ namely: (1) a sonographic transducer with built-in needle slots; (2) a transducer equipped with an attachable stretcher guide; and (3) a free hand approach, viewing through a transducer without a guide. The built-in needle slot ultrasound transducer is more suitable for guiding transthoracic biopsy as has been discussed previously. ${ }^{11}$

Since the colour Doppler puncture guiding device provides better real time control of the position of the needle and the identification of location and number of vessels, an improvement in safety is expected. However, the absence of any complications in this study does not allow us to conclude that the colour Doppler puncture guiding device is, in fact, safer than the grey scale device. A larger controlled comparative study is needed to verify this point.

\footnotetext{
1 Sanders RC. Percutaneous puncture: a new radiological skill. Radiology 1978;127:833-4.

2 Yang PC, Luh KT, Sheu JC, Kuo SH, Yang SP. Peripheral pulmonary lesions: ultrasonography and ultrasonically guided aspiration biopsy. Radiology 1985;155:451-6.

3 Yu CJ, Yang PC, Chang DB, Wu HD, Lee LN, Lee YC, et al. Evaluation of ultrasound-guided biopsies of mediastinal tumors: biopsy under ultrasound guidance. Chest 1991 100:399-405.

4 Wernecke K, Vassallo P, Peters PE, Von Bassewitz DB. Mediastinal tumors: biopsy under US guidance. Radiology 1989;172:473-6.

5 Charboneau JW, Reading CC, Welch TJ. CT and sonographically guided needle biopsy: current techniques and new innovations. $A \ngtr R \quad 1990 ; 154: 1-10$.

6 Ikezoe J, Morimoto S, Arisawa J, Takashima S, Kozuka T, Nakahara K. Percutaneous biopsy of thoracic lesion: value of sonography for needle guidance. AfR 1990;154:1181-5.
} 
7 Yang PC, Luh KT, Wu HD, Chang DB, Lee LN, Kuo SH, et al. Lung tumors associated with obstructive pneumonitis: US studies. Radiology 1990;174:717-20.

8 Ikezoe J, Sone S, Higashihara T, Morimoto S, Arisawa J, Kuriyama K. Sonographically guided needle biopsy for diagnosis of thoracic lesion. $A f R$ 1984;143:229-34.

9 Yang PC, Chang DB, Lee YC, Yu CJ, Kuo SH, Luh KT. Mediastinal malignancy: ultrasound guided biopsy through the supraclavicular approach. Thorax 1992;47: through

10 Chandrasekhar AJ, Reynes CJ, Churchill RJ. Ultrasonically guided percutaneous biopsy of peripheral pulmonary masses. Chest 1976;70:627-30.

11 Yang PC, Chang DB, Yu CJ, Lee YC, Wu HD, Kuo SH, Luh KT. Ultrasound-guided core biopsy of thoracic tumors. Am Rev Respir Dis 1992;146:763-7.

12 Petersen OM, Aasen TB, Gulsvik A. Fine needle aspiration biopsy of mediastinal and peripheral pulmonary masses guided by real-time sonography. Chest 1986;89:504-8.

13 Yuan A, Yang PC, Chang DB, Yu CJ, Lee YC, Kuo SH, Luh KT. Ultrasound-guided aspiration biopsy of small peripheral pulmonary nodules. Chest 1992;101:926-30.

14 Herman SJ, Holub RV, Weisbrod GL, Chamberlain DW. Anterior mediastinal masses: utility of transthoracic needle biopsy. Radiology 1991;180:167-70.

15 Yuan A. Chang DB, Yu CJ, Kuo SH, Luh KT, Yang PC. Color Doppler sonography of benign and malignant pC. Color Doppler sonography of benign

16 Kurohiji T, Sigel B, Justin J, Machi J. Motion marking in color Doppler ultrasound needle and catheter visualization. $\mathcal{F}$ Ultrasound Med 1990;9:243-5.
17 Hamper UM, Savader BL, Sheth S. Improved needle-tip visualization by color Doppler sonography. $A \mathcal{R} R$ 1991; 156:401-2.

18 Itoh K, Yamanaka T, Kasahara K, Koike M, Nakamura A, Hayaski A. Definitive diagnosis of pancreatic carcinoma with percutaneous fine needle aspiration biopsy under ultrasonic guidance. Am $\mathcal{F}$ Gastroenterol 1979;71: 469-72.

19 Hancke S, Holm HH, Koch F. Ultrasonically guided percutaneous fine needle biopsy of the pancreas. Surg Gynecol Obstet 1975;140:361-4.

20 Rasmusen SN, Holm HH, Kristensen JK, Barlebo $\mathrm{H}$. Ultrasonically guided liver biopsy. $B M \mathcal{Y}$ 1972;3:500-2.

21 Lee CH, Lan RS, Tsai YH, Chiang YC, Wang WJ. Riu's stain in the diagnosis of pulmonary cryptococcosis. Chest 1988;93:467-70.

22 Grant EG, Richardson JD, Smirnitopoulos JG, Jacobs NM. Fine needle biopsy directed by real-time sonography: tech-

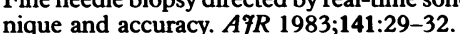

23 Izumi S, Tamaki S, Natori H, Kira S. Ultrasonically guided aspiration needle biopsy in disease of the chest. Am Rev Respir Dis 1982;125:460-4.

24 Mcgahan JP. Laboratory assessment of ultrasonic needle and catheter visualization. F Ultrasound Med 1986;5:373-7.

25 Heckemann R, Seidel KJ. The sonographic appearance and contrast enhancement of puncture needles. $\mathcal{f}$ Clin Ultrasound 1983;11:265-8.

26 Taylor KJW, Holland S. Doppler US Part I. Basic principles, instrumentation, and pitfalls. Radiology 1990;174:297307. 